\title{
VIT1/FBXO11 knockdown induces morphological alterations and apoptosis in B10BR mouse melanocytes
}

\author{
YONGWEI LI ${ }^{1}$, FEIFEI CHEN ${ }^{1}$, FUQUAN LIN ${ }^{1}$, CUIPING GUAN ${ }^{1}$, \\ XIAODONG WEI ${ }^{1}$, YINSHENG WAN ${ }^{2}$ and AIE XU ${ }^{1}$ \\ ${ }^{1}$ Department of Dermatology, the Third People's Hospital of Hangzhou, Hangzhou 310009, China; \\ ${ }^{2}$ Department of Biology, Providence College, Providence, RI 02918, USA
}

Received December 2, 2008; Accepted February 5, 2009

DOI: 10.3892/ijmm_00000179

\begin{abstract}
It has been shown that the VIT1 gene is downregulated in vitiligo melanocytes, but the expression of the VIT1 gene in melanocytes of Chinese patients with vitiligo and the potential function of VIT1 in pathogenesis of vitiligo are not as well known. In this study, we first compared VIT1 gene expression in melanocytes cultured from non-lesional skin of generalized vitiligo patients, normal melanocytes and melanomas in China. The cloned VIT1 ORF cDNA fragments were sequenced and then compared. VIT1 siRNAs were transfected into mouse B10BR melanocytes. The results from semi-quantitative RT-PCR demonstrated that the expression levels of VIT1 in non-lesional melanocytes from vitiligo patients are significantly lower than those in normal melanocytes. In contrast, the expression levels of VIT1 in melanoma are higher. The results also demonstrated that VIT1 is not a novel gene including a fragment of $81 \mathrm{bp}$ flanking by consensus GT and AG sequences on the $5^{\prime}$ and 3 ' ends, which is regarded as an intron. VIT1 without the extra intron is a known gene called FBXO11. Our findings revealed abnormal morphology by light and electron microscopes in FBXO11 siRNA transfected melancytes, which display large epithelioid perikaryon and stubby dendrites with occasional multidendricity as opposed to slender perikaryon and bipolar dendrites of controls. The electron microscopic observation indicated swelling mitochondria and endoplasmic reticulum (ER), and increased lysosomes and bubbles. Further, transfection of FBXO11 siRNA significantly promoted cell apoptosis. Collectively, this study provides comprehensive morphological proof of the relationship between dilation of ER and decreased levels of the FBXO11 gene in vitiligo melanocytes.
\end{abstract}

Correspondence to: Dr Aie Xu, Department of Dermatology, the Third People's Hospital of Hangzhou, Hangzhou 310009, P.R. China E-mail: xuaiehz@msn.com

Dr Yinsheng Wan, Department of Biology, Providence College, Providence, RI02918, USA

E-mail: yswan@providence.edu

Key words: VIT1/FBXO11, vitiligo, melanocyte, depigmentation

\section{Introduction}

Vitiligo is a common depigmentary disorder of the skin and hair and results from a selective destruction of melanocytes. It affects $\sim 0.5-2 \%$ of the world population without preference for specific skin tone or gender (1). Melanocytes, the pigmentproducing cells of the skin, are lost from vitiligo lesions (2). Pigment cells are not found within lesions either by morphologic criteria or through detection by antibodies commonly recognizing melanocytes. The consensus now is that the etiology of vitiligo involves a process of the loss of pigment cells rather than inhibition of melanogenesis.

It was shown that melanocytes from these vitiligo patients display morphologic and physiologic abnormalities. Specifically, melanocytes from vitiligo patients cultured under limiting conditions express a lag time in growth and are not subcultured. Moreover, melanocytes from normally pigmented vitiligo skin display melanosome compartmentalization and dilated endoplasmic reticulum (ER) profiles in vitro as well as in situ, in humans and animal models with the skin disorder $(3,4)$.

Vitiligo is a multigenic disorder presenting progressive depigmentation of the skin. The genetics of vitiligo cannot be explained by simple Mendelian genetics; it is characterized by imcomplete penetrance, multiple susceptibility loci and genetic heterogeneity. In recent years, linkage and association studies also provided strong support for vitiligo susceptibility genes on chromosomes 4q13-q21, 1p31, 7q22, 8p12 and 17p13 (5-7).

These genes underlying sensitivity to melanocyte loss remain an enigma. VIT1, a novel gene, is reportedly downregulated in vitiligo melanocytes. The 3 '-end of the VIT cDNA sequence is complementary to the 3 '-end of hMSH6, a $\mathrm{G} / \mathrm{T}$ mismatch repair gene. Preliminary results indicate that decreased levels of VIT1 are associated with increased levels of hMSH6 (2). However, the expression of the VIT1 gene in melanocytes of Chinese patients with vitiligo and potential function in pathogenesis of vitiligo remain unknown.

Given the potential role of VIT1 in the pathogenesis of vitiligo, we undertook this study to first compare the VIT1 expression in melanocytes cultured from non-lesional skin of generalized vitiligo patients, normal and melanomas and then transfect VIT1 siRNA into the melanocyte cell line B10BR to further study some potential functions of this gene in the pathogenesis of vitiligo. 


\section{Materials and methods}

Cell culture. Melanocytes were isolated from non-lesional skin tissue of patients diagnosed with generalized vitiligo as well as from skin of control individuals. Melanocytes were isolated from blister suction material or from full-thickness foreskin as control. From full-thickness foreskin, melanocytes were isolated by treatment with $0.25 \%$ trypsin in phosphate-buffered saline (PBS) at $37^{\circ} \mathrm{C}$ after trimming off dermis by treatment with dispase at $4^{\circ} \mathrm{C}$ overnight. Normal human melanocytes were propagated in TICVA, the culture medium consisting of Ham's F10 (Gibco, USA) with 7\% fetal bovine serum (Gibco), 2 mM glutamine, $50 \mathrm{ng} / \mathrm{ml}$ phorbol 12-myristate 13-acetate (TPA), $0.1 \mathrm{mM} 3$-isobutyl-1-methyl xanthine (IBMX), $1.0 \mu \mathrm{M}$ $\mathrm{Na}_{3} \mathrm{VO}_{4}, 0.1 \mathrm{mM}$ dbcAMP, $2.5 \mathrm{nM}$ cholera toxin (all from Sigma, St. Louis, MO) and penicillin $100 \mathrm{U} / \mathrm{ml}$ plus streptomycin $100 \mu \mathrm{g} / \mathrm{ml}$. bFGF (10 ng/ml) and ET-1 (1 nM) were chosen to accommodate slow-growing vitiligo melanocytes. Contaminating fibroblasts were removed by treatment with $0.1 \mathrm{mg} / \mathrm{ml}$ geneticin for 3 consecutive days. Cells were routinely split $1: 2$ at $100 \%$ confluence.

B10BR mouse melanocytes were cultured in Hams F12 supplement with $7 \%$ heat-inactivated calf serum, $50 \mathrm{ng} / \mathrm{ml}$ phorbol 12-myristate 13-acetate (TPA) and 1\% penicillin/ streptomycin. Cells were maintained at $37^{\circ} \mathrm{C}$ in a saturated humidity atmosphere containing $95 \%$ air and $5 \% \mathrm{CO}_{2}$. Cells were routinely grown in $25-\mathrm{cm}^{2}$ culture flasks.

RNA isolation. At $80 \%$ confluency, RNA was isolated from cells propagated in $75-\mathrm{cm}^{2}$ flasks. To this end, adherent cells were washed in PBS. Total RNA was prepared using TRIReagent (Invitrogen, USA) and total RNA extracted in chloroform, ethanol precipitated and stored at $-80^{\circ} \mathrm{C}$.

$R T$-PCR . Expression of an open reading frame (ORF) was defined by detection of a 423-bp fragment following RTPCR amplification in presence of primers VIT1-F: 5'-GGAT CCATGAAAGAACAAAATAAT-3' and VIT1-R: 5'-GAA TTCTCAGTTGTG CTGCAATGT-3'. RT was carried out at $48^{\circ} \mathrm{C}$ for $50 \mathrm{~min}$ with $1-2 \mu \mathrm{g}$ of RNA in the presence of $1 \mathrm{mmol} / \mathrm{l}$ $\mathrm{MgSO}_{4}, 0.2 \mathrm{mmol} / \mathrm{l}$ nucleotides, $50 \mathrm{U} \mathrm{AMV}$, and $1 \mathrm{pmol} / \mathrm{l}$ reverse primer. PCR was performed in the presence of forward and reverse primers, $20 \mathrm{M}$ nucleotidesm, $4 \mathrm{U}$ Tfl DNA polymerase and $1 \times$ PCR buffer. PCR amplification was carried out as 40 cycles at $94^{\circ} \mathrm{C}$ for $30 \mathrm{sec}$, at $55^{\circ} \mathrm{C}$ for $45 \mathrm{sec}$, and at $68^{\circ} \mathrm{C}$ for $45 \mathrm{sec}$, followed by $10 \mathrm{~min}$ at $72^{\circ} \mathrm{C}$. The resulting PCR products were size fractionated on a $2 \%$ agarose gel in TAE for $45 \mathrm{~min}$ at $80 \mathrm{~V}$, together with a 100 nucleotide ladder. For semi-quantitative RT-PCR, RNA was reverse transcribed to investigate relative expression of VIT1 from non-lesional vitiligo melanocytes versus normal control. Primers used for amplification were 5'-CATTAAGAAGTGC CATCA-3' and 5'-GTTACAATG GCAGGACTT-3' (the size of fragment is $298 \mathrm{bp}$ ). RT-PCR was carried out using onestep method (Promega, USA) in presence of $50 \mathrm{U}$ for each AMV and Tfl DNA polymerase, $0.2 \mathrm{mmol} / 1 \mathrm{dNTPs}, 1 \mathrm{mmol} / \mathrm{l}$ $\mathrm{MgSO}_{4}, 1 \mathrm{pmol} / \mathrm{l}$ primers. Reverse transcription was performed at $48^{\circ} \mathrm{C}$ for $50 \mathrm{~min}, 94^{\circ} \mathrm{C}$ for $2 \mathrm{~min}$. Amplification was carried out as 35 cycles of $30 \mathrm{sec}$ at $94^{\circ} \mathrm{C}, 45 \mathrm{sec}$ at $55^{\circ} \mathrm{C}, 45 \mathrm{sec}$ at $68^{\circ} \mathrm{C}$, ending with a 10 min elongation step at $72^{\circ} \mathrm{C}$. Products were electrophoresed in a $2 \%$ agarose gel in TAE buffer in the presence of ethidium bromide.

Fragment cloning and sequence analysis. Fragments with nearly correct size were isolated from the gel and cloned into a Teasy-Vector. Plasmids were transformed into XL1-Blue and positive clones were recognized as white colonies on X-gal/IPTG-coated agar plates. Plasmids were re-isolated from bacteria grown in ampicillin to select for presence of the plasmid. Vector and fragments were separated by agarose gel electrophoresis, and the fragment was isolated using a gel extraction kit (Sangon, Shanghai, China). Cloned cDNA fragments of interest were sequenced by Sangon. The sequences generated were compared by BLAST search of databases containing GenBank sequences of previously identified genes.

siRNA preparation. siRNAs corresponding to FBXO11 were designed and chemically synthesized by Invitrogen Life Technologies. The following FBXO11 siRNA were successfully. siRNA1 sense (FBXO11-MSS212759): 5'-AGA UCUUGUUCUCUAUGAACUGUCC-3', siRNA1 antisense (FBXO11-MSS212759): 5'-GGACAGUUCAUAGAGAAC AAGAUCU-3'; siRNA2 sense (FBXO11-MSS212760): 5'AUAUACAGAUGCCACCAUCUCUUCC, siRNA2 antisense (FBXO11-MSS212760): 5'-GGAAGAGAUGGUGGC AUCUGUAUAU-3'; siRNA3 sense (FBXO11-MSS212761): 5'-AUGUGAACACACCAACAUCACGUCC-3', siRNA3 antisense (FBXO11-MSS212761): 5'-GGACGUGAUGUU GGUGUGUUCACAU-3'. Stealth ${ }^{\mathrm{TM}}$ RNAi negative control (Cat. No. 12935-200) was designed by Invitrogen.

Transfection experiments. Transfection of siRNA-FBXO11 was conducted with Lipofectamine ${ }^{\mathrm{TM}} 2000$ (Invitrogen, USA) in 6-well plates according to the manufacturer's specifications. Briefly, the day before transfection, the cells were trypsinized, counted, and seeded at $1 \times 10^{5}$ cells per well into 6-well plates, so that they were $50 \%$ confluent on the day of transfection. siRNAs (20 pmol) and Lipofectamine ${ }^{\mathrm{TM}} 2000$ were diluted in $50 \mu 1$ of Opti-MEM I Reduced Serum Medium without serum and mixed gently and incubated for $5 \mathrm{~min}$ at room temperature. After the 5 min incubation, the diluted siRNA was combined with the diluted Lipofectamine ${ }^{\mathrm{TM}}$ 2000. The mix was incubated for 20-30 min at room temperature to allow the siRNA:Lipofectamine ${ }^{\mathrm{TM}} 2000$ complexes to form. Complexes $(100 \mu \mathrm{l})$ were added to each well, and then incubated at $37^{\circ} \mathrm{C}$ in a $\mathrm{CO}_{2}$ incubator for $48 \mathrm{~h}$.

Apoptosis assay. Flow cytometry was used to detect apoptosis by Annexin V-FITC apoptosis detection kit (Bio Vision). Cells were detached by trypsin-EDTA (Gibco) after treatments and were then incubated in $500 \mu 1$ binding buffer, $5 \mu 1$ annexin V-FITC and propidium iodide of each at room temperature for $5 \mathrm{~min}$ in the dark. Cell apoptosis histograms were generated after analysis of PI-stained cells by FACS with a BectonDickinson FACScan.

Electron microscopy. Cells were detached by trypsin-EDTA (Gibco) after treatments and were then collected. They were fixed with $2.5 \%$ glutaraldehyde in $0.1 \mathrm{M}$ phosphate buffer at $4^{\circ} \mathrm{C}$ overnight and post-fixed with $1 \% \mathrm{OsO}_{4}$ in $0.1 \mathrm{M}$ phosphate 


\section{T-1 CHN FBXOl-1 V T 1}

VT-1 $1-\mathrm{AN}$
FBXOI1
V T 1
U T-1 1 aHN
FBXO1-1
UT 1
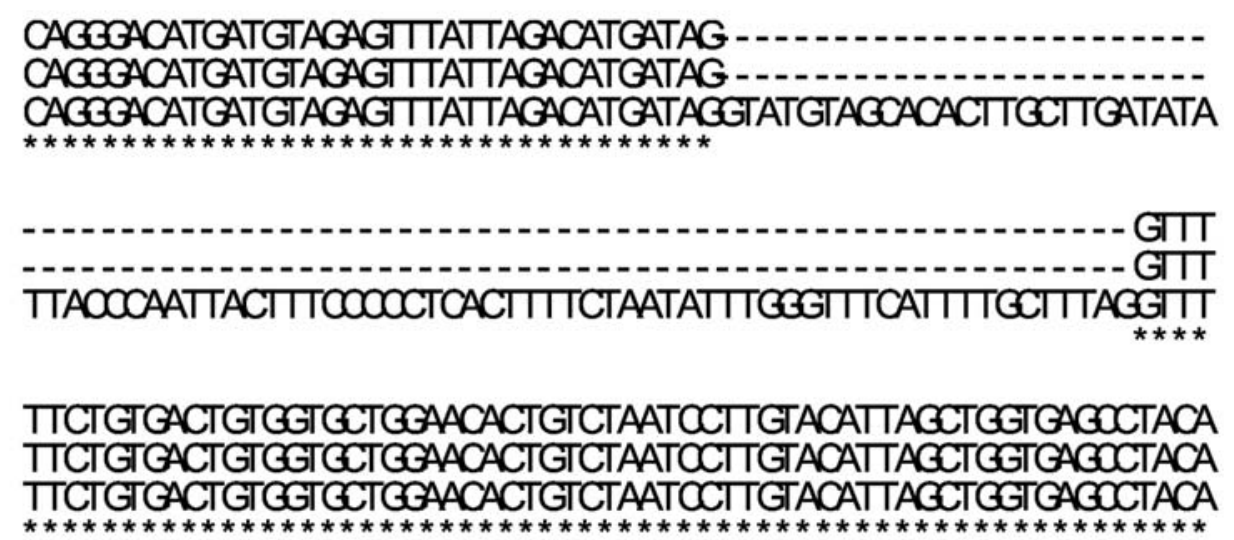

Figure 1. Homology between VIT-1_CHN, VIT1 (AF264714) and FBXO11 (NM_025133). buffer at $4^{\circ} \mathrm{C}$ for $1 \mathrm{~h}$. The cells were then embedded in $5 \%$ agarose which were then cut into 2-3 $\mathrm{mm}^{2}$ blocks, dehydrated in a graded series of ethanol and embedded in epoxy resin. Ultrathin sections were stained with uranyl acetate and lead citrate and were observed with an electron microscope (JEM1230) (JEOL, Tokyo, Japan).

Statistical analysis. Significance of differences was evaluated by two-sided Student's t-test. Mean differences were considered significant at $\mathrm{p}<0.05$.

\section{Results}

Confirmation of VIT1 ORF. One fragment including VIT1 ORF in non-lesional melanocytes cultured from Chinese vitiligo patients was amplified and sequenced. Homology between VIT-1_CHN, VIT1 (AF264714) and FBXO11 (NM_025133) is shown in Fig. 1. Except for one fragment of $81 \mathrm{bp}$, no difference of sequence was found between VIT1 and FBXO11. The fragment of $81 \mathrm{bp}$ is flanked by consensus GT and AG sequences on the 5' and 3 ' ends, supporting their identity as an intron.

Expression of FBXO11 in melanocytes. The expression level of FBXO11 mRNA was compared in melanocytes cultured from non-lesional skin of generalized vitiligo patients, normal and melanomas in China. The results showed that vitiligo melanocytes expressed diminished levels of FBXO11 mRNA compared with control samples (Fig. 2). We also found that melanomas expressed increased levels of FBXO11 mRNA compared with normal samples (data not shown).

FBXO11 siRNA knockdown affects the morphology of melanocytes. In order to study the effects of inhibition of FBXO11 gene expression on mouse immortalized B10BR melanocytes, three different siRNAs for FBXO11 were synthesized separately. The results obtained from semiquantitative RT-PCR revealed that siRNA1 inhibits FBXO11 expression markedly (Fig. 3A). siRNA also affects the differentiation of B10BR cells. B10BR melanocyte revealed unique morphological patterns under the light microscope after inhibition of FBXO11 expression for 2 days. They displayed large epithelioid perikaryon and stubby dendrite as

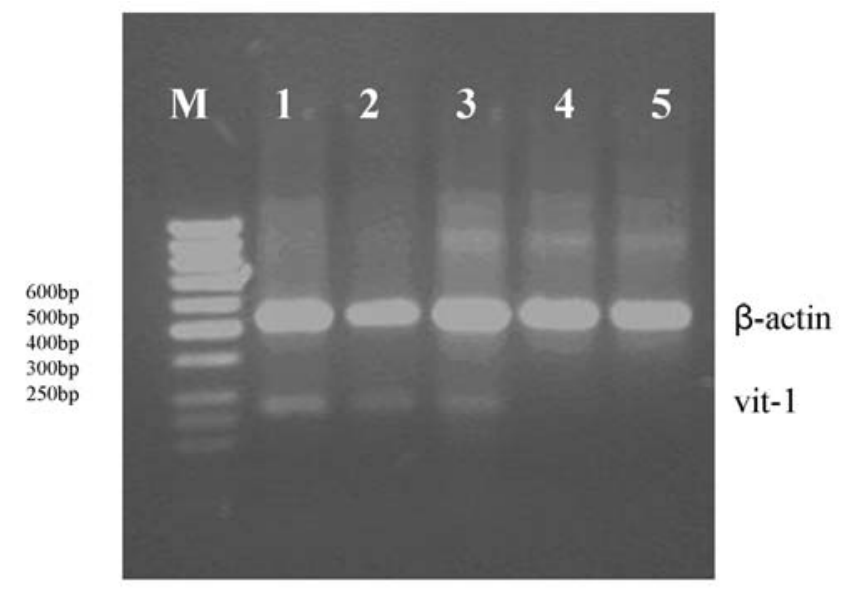

Figure 2. Expression of VIT1 in melanocyte with vitiligo. M: marker, 1, 2 and 3 are there normal controls, 4 and 5 are two patients with vitiligo. The length of amplified fragments of VIT1 observed upon agarose gel electrophoresis corresponds to the expected $298 \mathrm{bp}$, and the length of amplified fragments of ß-actin is $598 \mathrm{bp}$.

opposed to slender perikaryon and bipolar dendrites of the control cells (Fig. 3B). Electron microscopic observation indicated endoplastic reticulum retention (Fig. 4, upper panel) and increased lipid droplets (Fig. 4, lower panel) in B10BR melanocytes.

FBXO11 siRNA knockdown affects apoptosis in B10BR melanocytes. To further investigate the functions of FBXO11 in melanocytes, B10BR cells were cultured, transfected with FBXO11 siRNA and the apoptotic rate was measured by FACS analysis. The results showed that FBXO11 siRNA treated cells exhibit a higher apoptosis rate compared to control siRNA, with $35.8 \%$ of positive cells for annexin V-FITV in FBXO11 siRNA treated cells vs. $6.47 \%$ in controls.

\section{Discussion}

Genetic factors are suspected contributors of vitiligo etiopathogenesis. It was noted that the disease clusters in families, and that certain communities with high rates of consanguinial marriages display an increased incidence of the disease. 
A SIRNA1 SiRNA2 Neg CK

InnVit

G3PDH

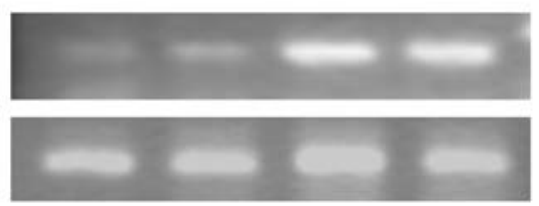

B

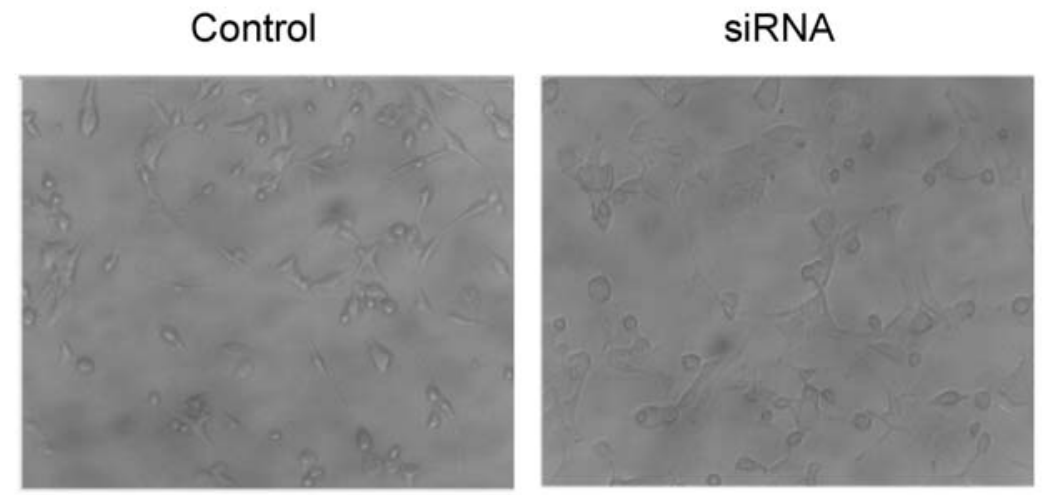

Figure 3. siRNA knockdown of FBXO11 affects cell differentiation in B10BR melanocytes. (A) B10BR cells were cultured and transfected with siRNAs for FBXO11, negative control siRNA and control only. RT-PCR was then performed as described in Materials and methods. (B) Differentiation of melanocytes in siRNA treated cultures. Phase contrast microscopy images were taken of melanocytes grown 2 days after transfection.

VIT1, a novel gene down-regulated in vitiligo melanocytes, is with the 3 '-end of the VIT1 cDNA sequence complementary to the 3'-end of hMSH6, a G/T mismatch repair gene. Preliminary results indicate that decreased levels of VIT1 are associated with increased level of hMSH6, while the functions of VIT1 remain elusive.

To investigate the expression of the VIT1 gene in melanocytes of Chinese patients with vitiligo, the gene expression was first compared in melanocytes cultured from non-lesional skin of generalized vitiligo patients (8 samples), normal (4 samples) and melanomas (2 samples). The cloned VIT1 ORF cDNA fragments were sequenced, and then compared. Semiquantitative RT-PCR results demonstrated that the expression levels of VIT1 in non-lesional melanocytes from vitiligo patients are significantly lower than those in normal melanocytes (Fig. 2). In contrast, the expression levels of VIT1 in melanoma were higher. Interestingly, we found that the cDNA sequence has one more $81 \mathrm{bp}$ fragment included in VIT1 ORF in normal and vitiligo melanocytes. This fragment containing the GT-AG splice site is a potential intron. Sequence analysis revealed that VIT1 actually is a part of FBXO11 belonging to the F-box protein family (Fig. 1).

The F-box protein constitutes one of the four subunits of ubiquitin protein ligase complex called SCF (SKP1-cullinF-box), which functions in phosphorylation-dependent ubiquitination. The SCF induces protein degradation, and the reduced SCF causes dilated ER resulting in a deficiency of melanogenesis. Our results support that notion (Fig. 4). Our findings also suggest that VIT1 is a part of an already defined gene. Future studies will hopefully consolidate our findings and lead to a better understanding of the role of this protein in pathogenesis of vitiligo.
The $\mathrm{N}$ terminus of FBXO11 contains the $\sim 40$ amino acid F-box motif common to all F-box protein family members $(2,4)$. FBXO11 also contains a zinc finger-like domain called a UBR box at the $\mathrm{C}$ terminus. The central region of FBXO11 contains a large domain with homology to the bacterial periplasmic protein NosD, a component of the nitrous oxide reductase complex (8). Overlapping with the NosD domain are three small carbohydrate-binding proteins and sugar hydrolases (CASH) domains of unclear function (9). FBXO11 is a member of the F-box protein subfamily lacking a distinct unifying domain (the O in FBXO11 stands for other) (10-12). The two other F-box subfamilies are FBXW, for proteins containing WD-40 repeat domains that are involved in phosphoprotein binding (13), and FBXL, for proteins containing leucine-rich repeat (LRR) domains, which also mediate protein-protein interactions (14). A few F-box proteins have been studied, among them are Skp2 (FBXL1), $\beta$-Trcp (FBXW1), Cdc4 (FBXW7). Structural studies suggest that the SCF complex maintains a C-shape structure with the substratebinding domain of the F-box protein at one end and the Roc1/E2 subunits at the other (15). It was shown that FBXO11 is a novel Nedd8 ligase for p53 and a component of the SCF complex, and that FBXO11 is an inhibitor of p53 transcripitonal activity (16). Our data shows that FBXO11 knockdown induces cell apoptosis (Fig. 5) which supports the above notion.

It was shown that melanocytes from these vitiligo patients display morphologic and physiologic abnormalities. Specifically, melanocytes from vitiligo patients cultured under limiting conditions expressed a lag time in growth and were not subcultured. Moreover, melanocytes from normally pigmented vitiligo skin displayed melanosome compartmentalization and dilated ER profiles in vitro as well as in situ. Dilated ER profile 

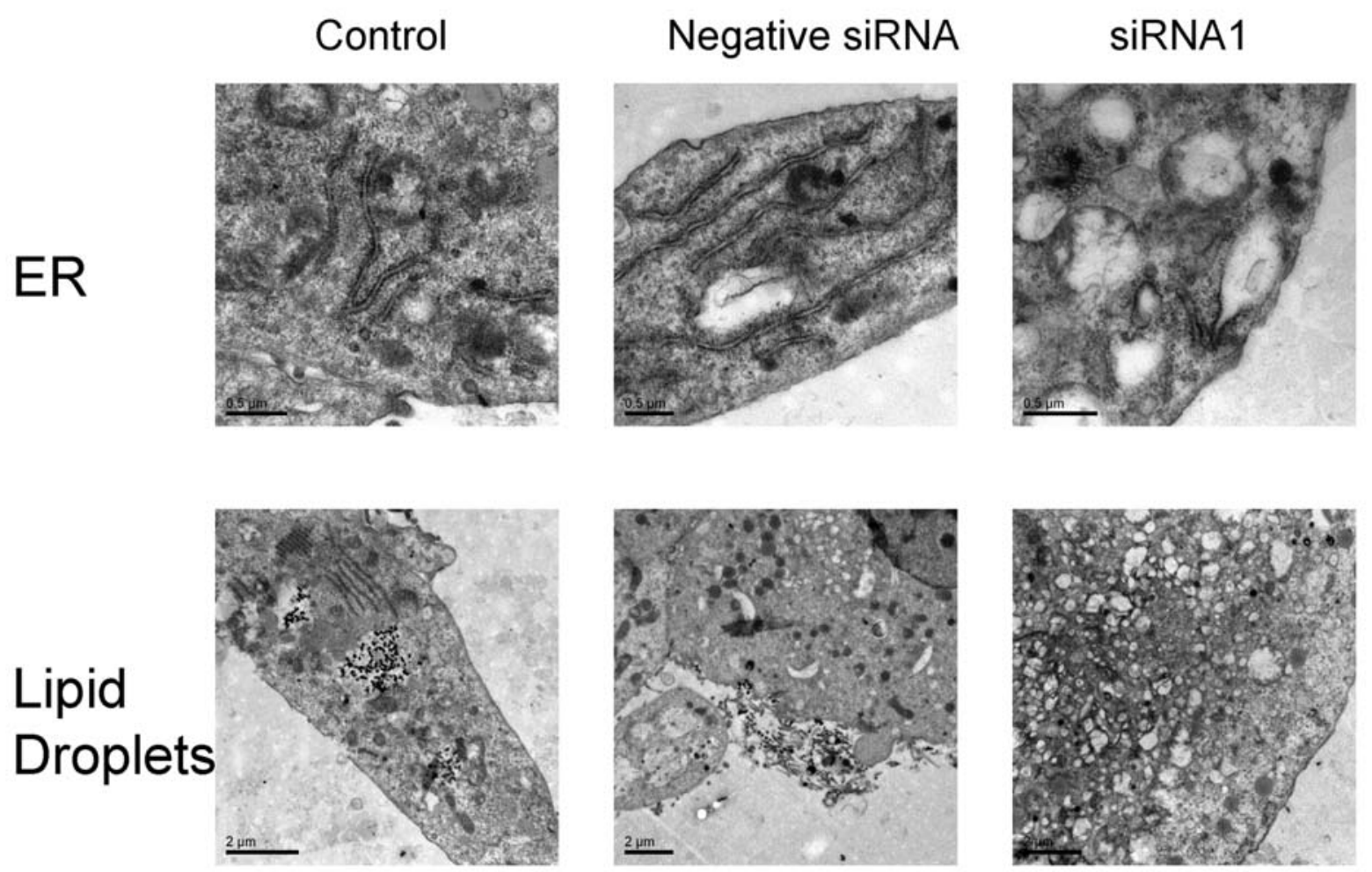

Figure 4. Ultrastructure alteration of B10BR melanocytes after knockdown of FBXO11 gene. Electron microscopic observation indicated endoplastic reticulum retention (upper panel), increased lipid droplets (lower panel) in B10BR melanocytes.
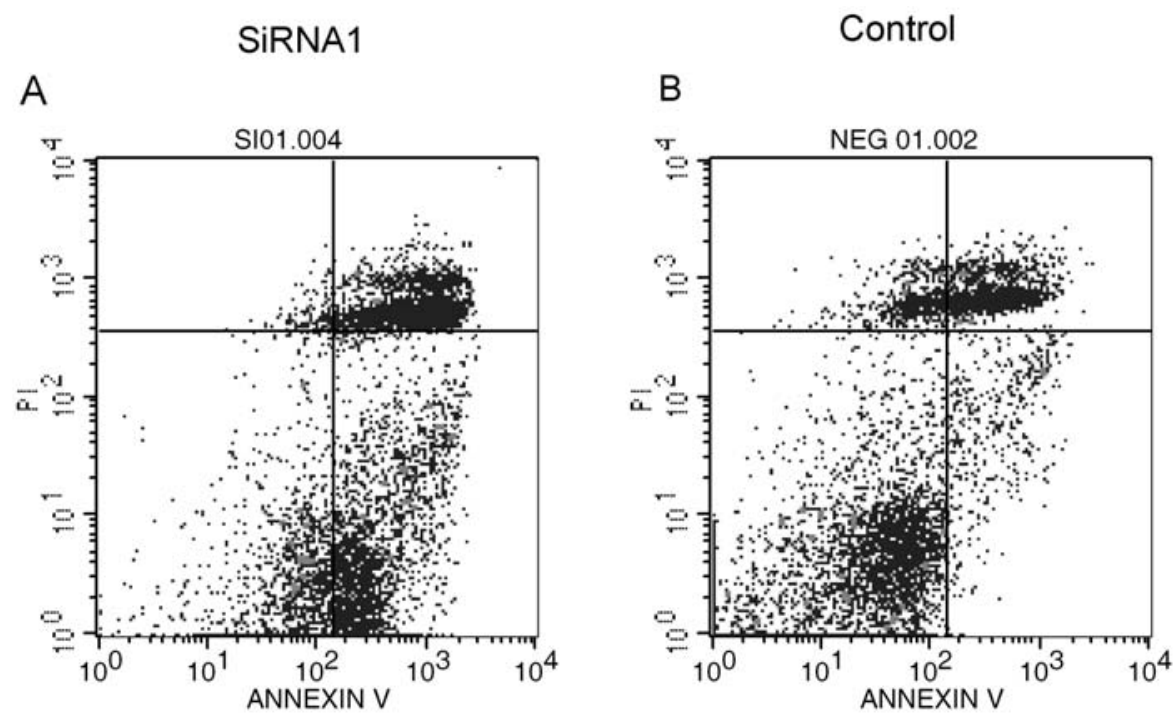

Figure 5. Knockdown of FBXO11 affects apoptosis of B10BR cells. B10BR cells were cultured and transfected with siRNA and apoptosis was detected by FACS analysis. A, siRNA1; B, Control.

shows protein accumulation in melanocytes of vitiligo patients. Our data also showed that inhibition of FBXO11 expression in B10BR melanocytes leads to dilated ER profiles just as vitiligo melanocytes (Fig. 4).

In summary, this study provides comprehensive morphological proof of the relationship between dilation of ER and decreased levels of the FBXO11 gene in vitiligo melanocytes.
Future studies will hopefully lead to a better understanding of the role of the FBXO11 gene in melanocytes of vitiligo patients.

\section{Acknowledgments}

The study was supported in part by grants from the National Natural Science Foundation of China (No. 30671896), the 
Natural Science Foundation of Zhejiang Province (Z206607) and NIH (P20 RR016457 from INBRE Program of the National Center for Research Resources).

\section{References}

1. Zhang XJ, Chen JJ and Liu JB: The genetic concept of vitiligo. J Dermatol Sci 39: 137-146, 2005.

2. Le Poole IC, van den Wijngaard RM, Westerhof W, Dutrieux RP and Das PK: Presence or absence of melanocytes in vitiligo lesions: an immunohistochemical investigation. J Invest Dermatol 100: 816-822, 1993

3. Boissy RE, Beato KE and Nordlund JJ: Dilated rough endoplasmic reticulum and premature death in melanocytes cultured from the vitiligo mouse. Am J Pathol 138: 1511-1525, 1991.

4. Boissy RE, Liu YY, Medrano EE and Nordlund JJ: Structural aberration of the rough endoplasmic reticulum and melanosome compartmentalization in long-term cultures of melanocytes from vitiligo patients. J Invest Dermatol 97: 395-404, 1991.

5. Zhang XJ, Liu JB, Gui JP, et al: Characteristics of genetic epidemiology and genetic models for vitiligo. J Am Acad Dermatol 51: 383-390, 2004.

6. Fain PR, Gowan K, LaBerge GS, et al: A genomewide screen for generalized vitiligo: confirmation of AIS1 on chromosome $1 \mathrm{p} 31$ and evidence for additional susceptibility loci. Am J Hum Genet 72: 1560-1564, 2003.

7. Alkhateeb A, Stetler GL, Old W, et al: Mapping of an autoimmunity susceptibility locus (AIS1) to chromosome 1p31.3p32.2. Hum Mol Genet 11: 661-667, 2002.
8. Zumft WG, Viebrock-Sambale A and Braun C: Nitrous oxide reductase from denitrifying Pseudomonas stutzeri. Genes for copper-processing and properties of the deduced products, including a new member of the family of ATP/GTP-binding proteins. Eur J Biochem 192: 591-599, 1990.

9. Ciccarelli FD, Doerks T and Bork P: AMOP, a protein module alternatively spliced in cancer cells. Trends Biochem Sci 27: 113-115, 2002.

10. Jin J, Cardozo T, Lovering RC, Elledge SJ, Pagano M and Harper JW: Systematic analysis and nomenclature of mammalian F-box proteins. Genes Dev 18: 2573-2580, 2004.

11. Winston JT, Koepp DM, Zhu C, Elledge SJ and Harper JW: A family of mammalian F-box proteins. Curr Biol 9: 1180-1182, 1999.

12. Cenciarelli C, Chiaur DS, Guardavaccaro D, Parks W, Vidal M and Pagano M: Identification of a family of human F-box proteins. Curr Biol 9: 1177-1179, 1999.

13. Smith TF, Gaitatzes C, Saxena K and Neer EJ: The WD repeat: a common architecture for diverse functions. Trends Biochem Sci 24: 181-185, 1999.

14. Enkhbayar P, Kamiya M, Osaki M, Matsumoto T and Matsushima N: Structural principles of leucine-rich repeat (LRR) proteins. Proteins 54: 394-403, 2004.

15. Zheng N, Schulman BA, Song L, et al: Structure of the Cul1Rbx1-Skp1-F boxSkp2 SCF ubiquitin ligase complex. Nature 416: 703-709, 2002

16. Abida WM, Nikolaev A, Zhao W, Zhang W and Gu W: FBXO11 promotes the Neddylation of p53 and inhibits its transcriptional activity. J Biol Chem 282: 1797-1804, 2007. 